\title{
AT-Hook-Containing Transcription Factor
}

National Cancer Institute

\section{Source}

National Cancer Institute. AT-Hook-Containing Transcription Factor. NCI Thesaurus.

Code C158844.

AT-hook-containing transcription factor (1439 aa, 155 kDa) is encoded by the human AKNA gene. This protein is involved in the positive regulation of expression of tumor necrosis factor receptor superfamily member 5 (CD40; TNFRSF5) and CD40 lig and. 